\title{
Complex Quadratic Bézier Curve on Unit Circle
}

\author{
Xuexiang Li and Junxiao Xue \\ School of Software, Zhengzhou University, Zhengzhou, China \\ Email: 1xx@zzu.edu.cn
}

\begin{abstract}
A Bézier curve is a parametric curve frequently used in computer graphics and related fields. In this paper, we firstly discusse the geometric and sudivision properties of the complex quadratic Bézier curve the on unit circle. Then, We discusse the relationship between the complex quadratic Bézier curve and Pascal spiral curves. Finally, we present the geometric continuity condition of composite curve.
\end{abstract}

Keywords - Bézier curve; Pascal spiral curve; geometric continuity

\section{INTRODUCTION}

Bézier curves are widely used in computer graphics to model smooth curves. As the curve is completely contained in the convex hull of its control points, the points can be graphically displayed and used to manipulate the curve intuitively. Affine transformations such as translation and rotation can be applied on the curve by applying the respective transform on the control points of the curve.

Quadratic and cubic Bézier curves are most common. Higher degree curves are more computationally expensive to evaluate. When more complex shapes are needed, low order Bézier curves are patched together, producing a Bézier spline. A Bézier spline is commonly referred to as a "path" in vector graphics standards (like SVG) and vector graphics programs (like Adobe Illustrator, CorelDraw and Inkscape). To guarantee smoothness, the control point at which two curves meet must be on the line between the two control points on either side.

The simplest method for scan converting (rasterizing) a Bézier curve is to evaluate it at many closely spaced points and scan convert the approximating sequence of line segments. However, this does not guarantee that the rasterized output looks sufficiently smooth, because the points may be spaced too far apart. Conversely it may generate too many points in areas where the curve is close to linear. A common adaptive method is recursive subdivision, in which a curve's control points are checked to see if the curve approximates a line segment to within a small tolerance. If not, the curve is subdivided parametrically into two segments, $0 \leqslant \mathrm{t} \leqslant 0.5$ and 0.5 $\leqslant \mathrm{t} \leqslant 1$, and the same procedure is applied recursively

Provincial Natural Science Foundation (132300410190) :Research on Digital Geometry Processing Technology and its Application Problem based on Conformal Geometry. to each half. There are also forward differencing methods but great care must be taken to analyse error propagation. Analytical methods where a spline is intersected with each scan line involve finding roots of cubic polynomials (for cubic splines) and dealing with multiple roots, so they are not often used in practice.

In animation applications, such as Adobe Flash and Synfig, Bézier curves are used to outline, for example, movement. Users outline the wanted path in Bézier curves, and the application creates the needed frames for the object to move along the path. For 3D animation Bézier curves are often used to define 3D paths as well as 2D curves for keyframe interpolation.

A Bézier curve is defined by a set of control points $\mathrm{P} 0$ through $\mathrm{Pn}$, where $\mathrm{n}$ is called its order $(\mathrm{n}=1$ for linear, 2 for quadratic, etc.). The first and last control points are always the end points of the curve; however, the intermediate control points (if any) generally do not lie on the curve.

Given points $\mathrm{P}_{0}$ and $\mathrm{P}_{1}$, a linear Bézier curve is simply a straight line between those two points. The curve is given by

$$
\begin{aligned}
B(t) & =p_{0}+t\left(p_{1}-p_{0}\right) \\
& =(1-t) p_{0}+t p_{1}, \quad t \in[0,1]
\end{aligned}
$$

and is equivalent to linear interpolation.

A quadratic Bézier curve is the path traced by the function $\mathrm{B}(\mathrm{t})$, given points $\mathrm{P}_{0}, \mathrm{P}_{1}$, and $\mathrm{P}_{2}$,

$$
\begin{array}{r}
B(t)=(1-t)\left[(1-t) p_{0}+t p_{1}\right]+t\left[(1-t) p_{1}+t p_{2}\right], \\
t \in[0,1]
\end{array}
$$

which can be interpreted as the linear interpolant of corresponding points on the linear Bézier curves from $\mathrm{P}_{0}$ to $\mathrm{P}_{1}$ and from $\mathrm{P}_{1}$ to $\mathrm{P}_{2}$ respectively. Rearranging the preceding equation yields:

$$
B(t)=(1-t)^{2}+t(1-t) p_{1}+t^{2} p_{2}, t \in[0,1] .
$$

The derivative of the Bézier curve with respect to $t$ is

$$
B^{\prime}(t)=2(1-t)\left(p_{1}-p_{0}\right)+2 t\left(p_{2}-p_{1}\right), t \in[0,1]
$$

from which it can be concluded that the tangents to the curve at $P_{0}$ and $P_{2}$ intersect at $P_{1}$. As $t$ increases from 0 to 1 , the curve departs from $\mathrm{P}_{0}$ in the direction of $\mathrm{P}_{1}$, then bends to arrive at $\mathrm{P}_{2}$ from the direction of $\mathrm{P}_{1}$.

The second derivative of the Bézier curve with respect to $t$ is

$$
B^{\prime \prime}(t)=2\left(p_{2}-2 p_{1}+p_{0}\right) .
$$


A quadratic Bézier curve is also a parabolic segment. As a parabola is a conic section, some sources refer to quadratic Béziers as conic arcs.

In this paper, we will discusse the geometric and sudivision properties of the complex quadratic Bézier curve on unit circle. Simultaneously We will discusse the relationship between the complex quadratic Bézier curve and Pascal spiral curves. Finally, the geometric continuity condition of composite curve will presented in the end.

\section{COMPLEX QUADRATIC BÉZIER CURVE}

\section{A. Definition}

Definition 2.1 Given three points $b_{0}, b_{1}$, and $b_{2}$, which are on the complex plane $\mathrm{C}$. The complex quadratic Bézier curve on the unit circle can be defined as

$$
\begin{aligned}
\mathrm{W}(\mathrm{t})= & \left(\frac{z_{1}-z}{z_{1}-z_{0}}\right)^{2} \mathrm{~b}_{0} \\
& +2\left(\frac{z_{1}-z}{z_{1}-z_{0}}\right)\left(\frac{z-z_{0}}{z_{1}-z_{0}}\right) \mathrm{b}_{1}, \\
& +\left(\frac{z-z_{0}}{z_{1}-z_{0}}\right)^{2} \mathrm{~b}_{2}
\end{aligned}
$$

where $\mathrm{z}=\mathrm{z}(\mathrm{t})$ is an unit circle (inferior) arc:

$$
\begin{aligned}
& \Gamma_{1}: \mathrm{z}(\mathrm{t})=e^{i t} \text {, } \\
& \theta_{0} \leq t \leq \theta_{1}, 0<=\theta_{1}-\theta_{0} \leq \pi,
\end{aligned}
$$

and $z_{0}=z\left(\theta_{0}\right), z_{1}=z\left(\theta_{1}\right)$, suppose that the counterclockwise central angle is positive direction.

We can get that

$$
\begin{aligned}
& \mathrm{W}\left(\theta_{0}\right)=\mathrm{b}_{0}, \mathrm{~W}\left(\theta_{1}\right)=\mathrm{b}_{1} ; \\
& W^{\prime}\left(\theta_{0}\right)=(\sin (\theta / 2))^{-1} \mathrm{e}^{-i \theta / 2}\left(\mathrm{~b}_{1}-\mathrm{b}_{0}\right),
\end{aligned}
$$

and

$$
W^{\prime}\left(\theta_{1}\right)=(\sin (\theta / 2))^{-1} \mathrm{e}^{i \theta / 2}\left(\mathrm{~b}_{2}-\mathrm{b}_{1}\right) 。
$$

\section{B. Geometric properties}

In order to analyze the singular inflection and convexity of the complex quadratic Bézier curve on the unit circle, we firstly analysis the properties of the general complex quadratic curves on unit circle.

$$
\omega(t)=d_{0}+d_{1} z+\frac{1}{2} d_{2} z^{2},
$$

where $d_{2} \neq 0, \quad z(t) \in \Gamma_{1}$.

$$
\begin{aligned}
& \text { Suppose } \mu=\frac{-d_{1}}{d_{2}}, \text { and rewrite curve (3) as } \\
& \omega=\omega(t, \mu)=d_{2}\left(\frac{1}{2} z^{2}-\mu z+\frac{d_{0}}{d_{2}}\right),
\end{aligned}
$$

Regar $t$ as the variable parameter of curve (4), then we can get the following theorem.

Theorem 2.1 Geometric properties of the unit circle on the complex two times curve is determined by the point locations in the complex plane (Figure 1).

(1) when $\mu \in \mathrm{N}$, it has no singularities and inflection points, it is a convex curve;

(2) when $\mu \in \mathrm{S}$, it has one and only one inflection point, and it has no singularity;

(3) when $\mu \in \mathrm{D}$, it has and only two inflection points, and it has no singularity;

(4) when $\mu \in \mathrm{C}$, it has one and only one cuspidal point, no focal points and inflection point;

(5) when $\mu \in \mathrm{L}$, it has two and only two key points, it has no inflection points and cuspidal points.

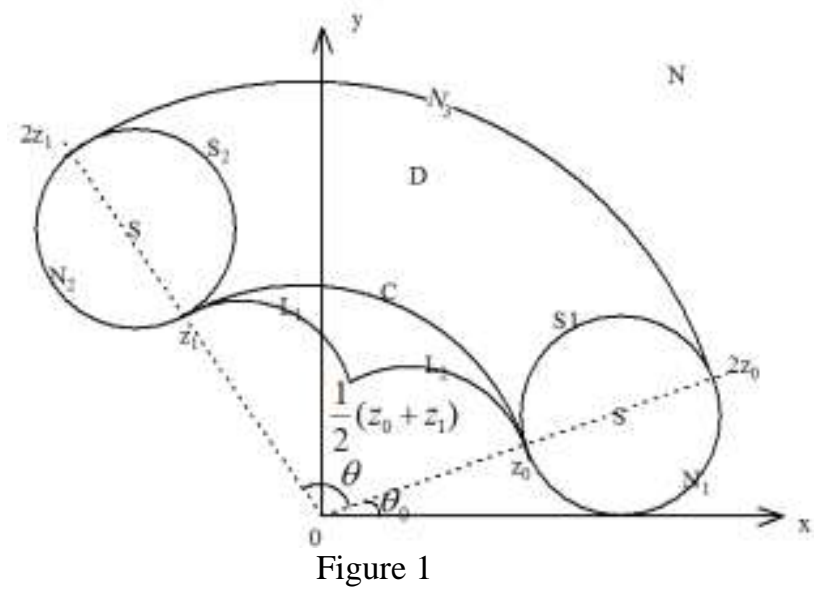

where the two disks of the region $\mathrm{S}$ are composed of $\operatorname{arc} S_{1}, N_{1}$ and $S_{2}, N_{2}$ surrounded by, contain $S_{1}$ and $\mathrm{S}_{2}$ but does not contain $N_{1}$ and $N_{2}$. Regional D surrounded by $\operatorname{arc} S_{1}, N_{3}, \mathrm{C}$ and $S_{2}$, but out of its own. Regional $\mathrm{L}$ surrounded $\mathrm{b}$ arc $\mathrm{C}, L_{1}$ and $L_{2}$, contain $L_{1}$ and $L_{2}$ but not contain $\mathrm{C}$. Regional $\mathrm{N}$ was about complex plane S, D, L and C complementary set. The curve of the following expression here:

$$
\begin{gathered}
\text { C: } \mu=e^{i t}, \quad \theta_{0}<t<\theta_{1}, \\
S_{1}: \mu=3 / 2 \bullet z_{0}+1 / 2 \bullet e^{i t}, \\
\theta_{0}<t<\pi+\theta_{0}, \\
S_{2}: \mu=3 / 2 \bullet z_{1}+1 / 2 \bullet e^{i t}, \\
\pi+\theta_{1}<t<2 \pi+\theta_{1}, \\
N_{1}: \mu=3 / 2 \bullet z_{0}+1 / 2 \bullet e^{i t}, \\
\pi+\theta_{0} \leq t \leq 2 \pi+\theta_{0}, \\
N_{2}: \mu=3 / 2 \bullet z_{1}+1 / 2 \bullet e^{i t}, \\
\theta_{1} \leq t \leq \pi+\theta_{1}, \\
N_{3}: \quad \mu=2 e^{i t}, \quad \theta_{0}<t<\theta_{1},
\end{gathered}
$$

where $z(t) \in \Gamma_{1}$. 
$L_{1}: \mu=1 / 2 \bullet z_{0}+1 / 2 \bullet e^{i t}, \quad \theta_{0}<t \leq \theta_{1}$,

$L_{2}: \mu=1 / 2 \bullet z_{1}+1 / 2 \bullet e^{i t}, \quad \theta_{0} \leq t<\theta_{1}$ 。

For complex two Bezier curve, we can get

$\mu=\left[z_{1} b_{0}-\left(z_{0}+z_{1}\right) b_{1}+z_{0} b_{2}\right] /\left[b_{0}-2 b_{1}+b_{2}\right]$ and

$b_{1}=\left[\left(z_{1}-\mu\right) b_{0}+\left(z_{0}-\mu\right) b_{2}\right] /$

$\left[\left(z_{1}-\mu\right)+\left(z_{0}-\mu\right)\right]$

Theorem 2.2 The unit circle (inferior) arc on the complex two Bezier $W_{1}\left(t ; b_{1}\right)$ geometric properties of curves is determined by the following location in the complex plane. When and only when the $b \in N, b_{1} \in S, b_{1} \in D, b_{1} \in C$ and $b_{1} \in L$, curve $W_{1}\left(t ; b_{1}\right)$ in order to convex curve, there is an inflection point, two point, with a sharp point and a two point(Figure 2). The expression of each curve is:

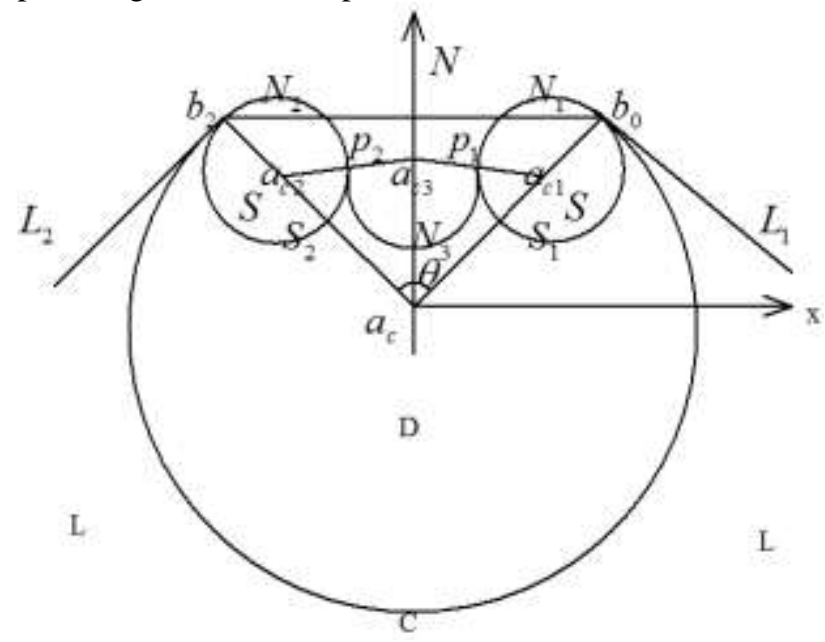

Figure 2

$C: v=a_{c}+\left|a_{c}-b_{0}\right| e^{i t},(\pi+\theta) / 2<t<(5 \pi-\theta) / 2$,

$a_{c}=\left(b_{0}+b_{2}\right) / 2+i \operatorname{ctg}(\theta / 2)\left(b_{2}-b_{0}\right) / 2 ;$

$S_{1}: v=a_{c 1}+1 / 4\left\lfloor\left|a_{c}-b_{0}\right| e^{i t}, \arg \left(p_{1}-a_{c 1}\right)<t<(5 \pi-\theta) / 2\right.$,

$a_{c 1}=b_{0}+\left(a_{c}-b_{0}\right) / 4, p_{1}=b_{0}+\left(b_{2}-b_{1}\right) z_{0} /\left(3 z_{0}-z_{1}\right)$

$S_{2}: v=a_{c 2}+1 / 4\left\lfloor\left|a_{c}-b_{2}\right| e^{i t},(\pi+\theta) / 2<t<\arg \left(p_{2}-a_{c 2}\right)\right.$,

$a_{c 2}=b_{0}+\left(a_{c}-b_{2}\right) / 4, p_{2}=b_{2}+\left(b_{2}-b_{0}\right) z_{1} /\left(z_{0}-3 z_{1}\right) ;$

$N_{1}: v=a_{c 1}+1 / 4\left\lfloor\left|a_{c}-b_{0}\right| e^{i t},(\pi-\theta) / 2 \leq t \leq \arg \left(p_{1}-a_{c 1}\right)\right.$
$N_{2}: v=a_{c 2}+1 / 4\left\lfloor\left|a_{c}-b_{2}\right| e^{i t}, \arg \left(p_{2}-a_{c 2}\right) \leq t \leq(\pi+\theta) / 2 ;\right.$

$N_{3}: v=a_{c 3}+\left|a_{c 3}-p_{1}\right| e^{i t}, \arg \left(a_{c 2}-p_{2}\right)<t<\arg \left(a_{c 1}-p_{1}\right) ;$

$L_{1}: v=b_{0}-u e^{-i \theta / 2}\left(b_{2}-b_{0}\right), u>0$

$L_{2}: v=b_{2}+u e^{i \theta / 2}\left(b_{2}-b_{0}\right), u>0$;

III RELATIONSHIP BETWEEN THE COMPLEX QUADRATIC BÉZIER CURVE AND PASCAL SPIRAL CURVES

Now, we discuss the relationship between the complex quadratic Bezier curve and Pascal spiral curves.

Theorem 3.1 Complex t quadratic Bezier curve in general:

$$
\begin{gathered}
\omega(t)=d_{0}+d_{1} z+\frac{1}{2} d_{2} z^{2}, \mathrm{z}(\mathrm{t}) \in \Gamma_{1}: \\
\mathrm{z}(\mathrm{t})=\mathrm{e}^{i t}, \theta_{0} \leq t \leq \theta_{1}, 0<\theta=\theta_{1}-\theta_{0} \leq \pi
\end{gathered}
$$

When and only when the

$\left(\operatorname{Re}\left(d_{2}\right)\right)^{2}-\left(\operatorname{Im}\left(d_{2}\right)\right)^{2}=\left(\operatorname{Re}\left(d_{1}\right)\right)^{2}-\left(\operatorname{Im}\left(d_{1}\right)\right)^{2}$,

$\omega(t)$ and Pascal equivalence. When and only when

the $d_{2}=0, \omega(t)$, arc and conical curve

equivalence. $\omega(t)$ and parabola was not equivalent.

Proof: For $\omega(t)=d_{0}+d_{1} z+\frac{1}{2} d_{2} z^{2}$,

set $d_{j}=a_{j}+i b_{j},(j=0,1,2), z=\cos t+i \sin t$, the $\omega(t)$ parameter equation of the form, That is:

$\left\{\begin{array}{l}x=a_{0}+a_{1} \cos t-b_{1} \sin t+\frac{1}{2}\left[a_{2} \cos 2 t-b_{2} \sin 2 t\right] \\ y=b_{0}+a_{1} \sin t+b_{1} \cos t+\frac{1}{2}\left[b_{2} \cos 2 t+a_{2} \sin 2 t\right]\end{array}\right.$

The parameter equation for Pascal spiral $\mathrm{C}(\mathrm{t})$.

$\left\{\begin{array}{l}x_{p}=(a \cos t+b) \cos t \\ y_{p}=(a \cos t+b) \sin t\end{array}\right.$

For $\omega(t)$ :

$\left\{\begin{array}{l}\hat{x}=x-a_{0} \\ \hat{y}=y-b_{0}\end{array}\right.$

Therefore, when and only when the $\left(\operatorname{Re}\left(d_{2}\right)\right)^{2}-\left(\operatorname{Im}\left(d_{2}\right)\right)^{2}=\left(\operatorname{Re}\left(d_{1}\right)\right)^{2}-\left(\operatorname{Im}\left(d_{1}\right)\right)^{2}$, The affine transformation between (5) and (6). 


$$
\left[\begin{array}{l}
\frac{a_{2}}{a} \frac{b_{2}-b_{1}}{b-a} \\
\frac{b_{2}}{a} \frac{-\left(a_{2}-a_{1}\right)}{b-a}
\end{array}\right]\left[\begin{array}{l}
x_{p} \\
y_{p}
\end{array}\right]+\left[\begin{array}{l}
-\frac{1}{2} a_{2} \\
-\frac{1}{2} b_{2}
\end{array}\right]=\left[\begin{array}{l}
\hat{x} \\
\hat{y}
\end{array}\right]
$$

So, $\omega(t)$ and $\mathrm{C}(\mathrm{t})$ equivalent.

The rest of the proposition can be proved similarly.

Theorem 3.2 Complex quadratic Bezier r curve.

$$
\begin{aligned}
& \mathrm{W}(t)=\left(\frac{z_{1}-z}{z_{1}-z_{0}}\right)^{2} \mathrm{~b}_{0}+2\left(\frac{z_{1}-z}{z_{1}-z_{0}}\right)\left(\frac{z-z_{0}}{z_{1}-z_{0}}\right) \mathrm{b}_{1}+ \\
& \left(\frac{z-z_{0}}{z_{1}-z_{0}}\right)^{2} \mathrm{~b}_{2}
\end{aligned}
$$

The $\mathrm{z}=\mathrm{z}(\mathrm{T})$ is the unit circle (inferior) arc

$$
\Gamma_{1}: \mathrm{z}(\mathrm{t})=\mathrm{e}^{i t}, \theta_{0} \leq t \leq \theta_{1}, 0<\theta=\theta_{1}-\theta_{0} \leq \pi,
$$

When and only when the:

$$
\begin{aligned}
& \left(\operatorname{Re}\left(b_{2}-2 b_{1}+b_{0}\right)\right)^{2}-\left(\operatorname{Im}\left(b_{2}-2 b_{1}+b_{0}\right)\right)^{2} \\
& =\left(\operatorname{Re}\left(z_{0} b_{2}-\left(z_{1}+z_{0}\right) b_{1}+z_{1} b_{0}\right)\right)^{2}-\left(\operatorname{Im}\left(z_{0} b_{2}-\left(z_{1}+z_{0}\right) b_{1}+z_{1} b_{0}\right)\right)^{2}
\end{aligned}
$$

$\mathrm{W}(\mathrm{t})$ equivalent of Pascal spiral;

When and only when: $b_{2}-2 b_{1}+b_{0}=0, \mathrm{~W}(\mathrm{t})$ and circular arc and conical curve equivalence. $\omega(t)$ cannot and parabolic equivalence.

Prove: General two degree complex curves and complex two Bezier curves have the following relationship.

$$
\left\{\begin{array}{l}
d_{0}=b_{0} z_{1}^{2}-2 z_{1} z_{0} b_{1}+z_{0}^{2} b_{2} \\
d_{1}=-2\left(z_{1} b_{0}-\left(z_{1}+z_{0}\right) b_{1}+z_{0} b_{2}\right) \\
d_{2}=2\left(b_{0}-2 b_{1}+b_{2}\right)
\end{array}\right.
$$

We can prove the above proposition by theorem 3.1 and the above formula.

\section{IVCONCLUSION}

With the above discussion, we can get that complex quadratic Bezier curves have limitations of relatively large, first of all, even the point of arc (with respect to the free parameter curves are not general representation of arc, has certain superiority),complex two Bezier curves for only the two time in from, essentially is a. In other words, if only to arc needs, using the properties of fractional linear mapping on complex field (the circular), when the variable is located on the circumference at, a line mapping that can express the circle arc, the point by proposition 3 can also see. Furthermore, due to complex two Bezier curve without segmentation of good property (mainly because of the complex two Bezier curve has no affine invariance), the practical application of partition property often need to use, should be said that good segmentation properties is carried out curve design must consider the question, so, in this respect, complex the two Bezier curves are also has great limitations.

\section{REFERENCES}

[1] D. S. Meek and D. J. Walton, The family of biarcs that matches planar, two-point G1 Hermite data[J], Journal of Computational and Applied Mathematics, 2008, 212(1): 31-45.

[2] J. W. Zhang, C-curves: an extension of cubic curves[J], Computer Aided Geometric Design,1996, 13(3): 199-217.

[3] Lv Yonggang, Wang Guozhao, and et al, Single arc spline shape preserving interpolation algorithm [J], Chinese Journal of computers, 2002, 25(5): 483-486.

[4] L. V. Ahlfors, Complex Alalysis[M], New York: McGrawHill Book Co., 1979.

[5] M. Yeung and D. J. Walton, Curve fitting with arc-splines for NC tool path generation[J], Computer-Aided Design, 1994, 26(11): 845-849.

[6] R.L. Scot Drysdale and G ${ }^{*}$ unter Rote and Astrid Sturm, Approximation of an open polygonal curve with a minimum number of circular arcs and biarcs[J], Computational Geometry: Theory and Applications[J], 2008, 41(1-2): 31-47.

[7] Shi Fazhong, Computer aided geometric design and non uniform rational B spline [M], Beijing: Beihang University press, 1994.

[8] Wang Qi, Guo Fei, Wang Qiyi, Circular arc spline approximation for auto programming profiles of machine parts [J], Chinese Journal of Mechanical Engineering, 1998 34(2): 20-25.

[9] Rachid Ait-Haddoua, Walter Herzog, Taishin Nomura Complex Bézier curves and the geometry of polygons, Computer Aided Geometric Design 27 (2010) 525-537.

[10] J. Sánchez-Reyes, Complex rational Bézier curves, Computer Aided Geometric Design, 26(8), 2009: 865-876 